\title{
Customização em massa da mídia: Veículos tradicionais do conhecimento se reinventam para atender os consumidores e coexistir com as novas mídias
}

Valdenise Schmitt*

Artigo recebido em: 20 de setembro de 2010

Aprovado em:

8 de outubro de 2010

* Doutoranda do Programa PósGraduação em

Engenharia e Gestão do Conhecimento (UFSC). Mestre em Engenharia e Gestão do Conhecimento.

Especialista em

Desenvolvimento de Aplicações Web.

Especialista em Novas Mídias, Rádio e TV.

val.schmitt@gmail.com

Abstract: The technological advances force the media and the vehicles of knowledge to continually mold themselves as survival alternatives. This article deals with changes in communication toward individualized news. It not only presents theories and models for the media life-cycle, but also provides examples of vehicles that are reinventing themselves and exploring new audience niches in the Information and Knowledge Age, when the overwhelming information, seen before as valuable, imposes strategies for the delivery of personalized content. It's concluded that the only guaranteed continuation of the vehicles of knowledge's life is the constant change in order to both satisfy and keep customers and earn profits.

Keywords: Media; innovation; individualization.

Resumo: Os avanços tecnológicos obrigam as mídias e os veículos do conhecimento a se modelarem continuamente como alternativa de sobrevivência. Este artigo trata de mudanças na comunicação em direção às notícias individualizadas. Além de apresentar teorias e modelos de ciclo de vida das mídias, oferece exemplos de veículos que estão se reinventando e explorando novos nichos de audiência na Era da Informação e do Conhecimento, em que a sobrecarga de informação, antes vista como um valor, agora impõe estratégias de distribuição individualizada de conteúdo. Conclui-se que a única previsão garantida de vida para os veículos do conhecimento é a constante mudança, para satisfazer e fidelizar os consumidores e auferir lucros.

Palavras-chave: Mídia; inovação; individualização.

\section{Mass customization of media:}

\section{Traditional vehicles of knowledge reinvente themselves} to serve the customer and co-exist with the new medias 


\section{Introdução}

$\mathrm{D}$ esde o século XX, as tecnologias de informação e comunicação vêm sofrendo rápidas e sucessivas inovações e, consequentemente, alterando o cenário comunicacional. A primeira descoberta científica que permitiu avanços significativos na comunicação foi o transistor, inventado em 1947, por John Bardeen, Walter H. Brattain e William Bradford Shockley, então pesquisadores dos Laboratórios Bell. Um pouco mais de duas décadas depois, em setembro de 1969, a criação da internet e seu desenvolvimento revolucionaram o campo comunicacional ao permitir que a estrutura da rede fosse utilizada como plataforma pelas mídias existentes (CASTELLS, 2003; LEHMAN-WILZIG; COHEN-AVIGDOR, 2004) e como meio econômico de disseminar mensagens para pequenas audiências (LEHMAN-WILZIG; COHEN-AVIGDOR, 2004). Tais potencialidades fizeram com que a internet fosse considerada tanto uma metamídia (metamedium) como uma macromídia (macromedium), compreensiva em escopo e global em tamanho (LEHMAN-WILZIG; COHEN-AVIGDOR, 2004).

Desde os anos 1990, a aplicação combinada entre as tecnologias de informática, telecomunicações e transmissão por redes digitais de comunicação apontam previsões e tendências, tais como o desaparecimento das mídias tradicionais, em especial das mídias impressas, e a morte dos suportes individuais (SAAD, 2003). A história mostra, com raras exceções, que o aparecimento de uma nova mídia não implica o desaparecimento das mídias existentes (BRIGGS; BURKE, 2006; LEHMAN-WILZIG; COHEN-AVIGDOR, 2004). Elas coexistem, tanto uma como outra passam por adaptações para garantir seu público e espaço no mercado (BRIGGS; BURKE, 2006; LEHMAN-WILZIG; COHEN-AVIGDOR, 2004). Exemplo mais recente são os jornais impressos e on-line que há mais de uma década e meia ${ }^{1}$ seguem se reinventando e evoluindo.

É fato que a ameaça de obsolescência sempre existiu, assim como a luta contínua para atrair (novos) e manter (antigos) consumidores. No momento, profissionais e empresários de mídias e veículos do conhecimento ${ }^{2}$ visualizam alternativas de sobrevivência diante da constante inovação digital, entre as quais, menciona-se a entrega de notícias segundo os gostos e necessidades dos consumidores (VANDEVANTER, 2009a, 2009b).

Este artigo, baseado nos modelos do ciclo de vida das mídias, mostra que as mídias e os veículos do conhecimento se reinventam e exploram novos nichos de audiência para prosperar e manter a competitividade na Era da Informação e do Conhecimento. $\mathrm{O}$ artigo trata de mudanças na comunicação em direção à customização em massa da mídia (notícias e publicidade segundo as pre-

\footnotetext{
${ }^{1}$ Apesar de algumas tentativas anteriores de distribuir notícias via internet, o primeiro jornal a migrar para a rede foi o San José Mercury News em 1993. No Brasil, o primeiro jornal a publicar uma versão eletrônica foi o Jornal do Brasil, em 28 de maio de 1995 (SCHMITT, 2003).

${ }^{2}$ Machlup (1962) denomina as indústrias de comunicação de indústrias do conhecimento, pois elas, assim como as entidades educacionais e de pesquisa e desenvolvimento produzem e reproduzem conhecimento na sociedade. Produzir conhecimento para o autor, não é somente descobrir, inventar, criar, planejar, é também disseminar e comunicar (MACHLUP, 1962). Neste sentido, é que são utilizadas as expressões mídias e veículos do conhecimento neste artigo. A afirmação de Meditsch (1997) de que o jornalismo produz e reproduz conhecimento reforça tal posicionamento.
} 
ferências dos consumidores) (VANDEVANTER, 2009a, 2009b) e apresenta exemplos de produtos individualizados.

A seção a seguir, que teve como fonte principal o trabalho de Lehman-Wilzig e Cohen-Avigdor (2004), traz teorias e modelos de desenvolvimento das mídias, inclusive o modelo de ciclo de vida das mídias elaborado pelos autores que, em seu estágio de adaptação, contempla a personalização ou customização de conteúdo (SCHMITT; OLIVEIRA, 2009) como uma possibilidade de resistência defensiva das mídias.

\section{Teorias e modelos de desenvolvimento das mídias}

Algumas teorias e modelos tentam explicar as fases de vida das mídias (LEHMAN-WILZIG; COHEN-AVIGDOR, 2004), dos meios de comunicação utilizados ao longo da história pelo homem para armazenar e transportar conhecimento (STANOEVSKA-SLABEVA, 2002).

Segundo Lehman-Wilzig e Cohen-Avigdor (2004), o primeiro modelo de desenvolvimento das mídias foi elaborado por John C. Merril e Ralph L. Lowenstein no início dos anos 1970. Baseado no tipo de audiência (elite, popular

\section{Profissionais,} empresários e veículos visualizam alternativas de sobrevivência diante da inovação digital e especializada), tal modelo mostra que a adoção de uma nova mídia inicia pelas classes mais altas da sociedade. Na sequência, o público em geral adota a mídia e, por último, as subaudiências, que fazem uso da mídia de maneira especializada. Gradativamente, segundo este modelo, as mídias vão sendo incorporadas pela sociedade (LEHMAN-WILZIG; COHEN-AVIGDOR, 2004). Observa-se que o modelo de Merril e Lowenstein é bastante sustentável nos dias atuais, pois quando novas mídias surgem, elas aparecem com preços sobrevalorizados, o que impede a aquisição da mídia pelas camadas menos favorecidas da sociedade, em um primeiro momento. À medida que os preços diminuem, a penetração aumenta.

A partir dos anos 1990 aparecem várias teorias e modelos de desenvolvimento das mídias, entre as quais de Donald Shaw (1991), Paul Saffo (1992), Dan Caspi (1993), Philip M. Napoli (1998), Maggie O’Brien (1999), Hanna Adoni e Hillel Nossek (2001) e de Lehman-Wilzig e Cohen-Avigdor (2004) (LEHMAN-WILZIG; COHEN-AVIGDOR, 2004).

De acordo com Lehman-Wilzig e Cohen-Avigdor (2004), em 1991, Shaw apresentou um modelo baseado nos estágios da vida humana - juventude, maturidade e velhice - para explicar o desenvolvimento das mídias. Nos três estágios, sugeria respostas criativas dos líderes para avanços tecnológicos (LEHMAN-WILZIG; COHEN-AVIGDOR, 2004).

Um ano mais tarde, Paul Saffo, baseado nos estudos que Everett Rogers fez por volta dos anos 1980, discute o elemento tempo no desenvolvimento das mídias (LEHMAN-WILZIG; COHEN-AVIGDOR, 2004; FIDLER, 1998). Para Saffo, 30 anos é o tempo necessário para que se introduzam novas ideias ou tecnologias em uma cultura e para que essas tenham completa adoção pelo público (FIDLER, 1998; LEHMAN-WILZIG; COHEN-AVIGDOR, 2004). Conforme a regra de 30 anos proposta e defendida por Saffo, na primeira década, não existe muita penetração no mercado da nova mídia, apesar de existir 
muito entusiasmo e confusão; na segunda, o produto começa a penetrar na sociedade; e, na terceira, ele é uma tecnologia comum que todos têm e usam (FIDLER, 1998; LEHMAN-WILZIG; COHEN-AVIGDOR, 2004).

Em 1993 é publicado o modelo de mídia formulado por Caspi. Neste modelo, o desenvolvimento da mídia é dividido em quatro estágios: inauguração, institucionalização, defesa e adaptação. De acordo com tal modelo, no primeiro estágio a nova mídia ganha muita atenção do público; no segundo, grande parte dos indivíduos adota a mídia; no terceiro, a hegemonia da mídia é ameaçada pelo aparecimento de uma outra mídia, mais nova; e, no último, a mídia antiga se adapta para coexistir com a mídia mais nova (LEHMAN-WILZIG; COHEN-AVIGDOR, 2004).

Napoli, em 1998, discute o estágio defensivo da mídia existente. Seu modelo de desenvolvimento apresenta quatro estágios: complacência, resistência (retórica, legal e econômica), diferenciação e diversificação (LEHMANWILZIG e COHEN-AVIGDOR, 2004). No ano seguinte, Maggie O'Brien apresenta possibilidades que a sociedade espera que ocorra com a velha mídia, a saber, (1) "mídiamorfose": adaptação e transformação; e (2) "mídiaciclo": morte (LEHMAN-WILZIG e COHEN-AVIGDOR, 2004).

Em 2001, Hanna Adoni e Hillel Nossek colocam a convergência das mídias, entendida como "fluxo de conteúdo através de múltiplos suportes midiáticos" (JENKINS, 2008, p. 27) dentro do estágio de resistência defensiva da mídia, oferecendo três possibilidades de interação: (1) equivalência funcional: a velha mídia é substituída pela nova; (2) diferenciação funcional: as mídias (velha e nova) encontram um modo de coexistir; (3) multiplicidade funcional: ambas se fundem em uma unidade multifuncional (LEHMAN-WILZIG e COHENAVIGDOR, 2004).

Fundamentado nos estudos dos autores mencionados, Lehman-Wilzig e Cohen-Avigdor (2004) elaboram um modelo que contempla seis estágios: (1) nascimento, (2) penetração, (3) crescimento, (4) maturação, (5) resistência defensiva e (6) adaptação, convergência e obsolescência, conforme mostra a Figura 1:

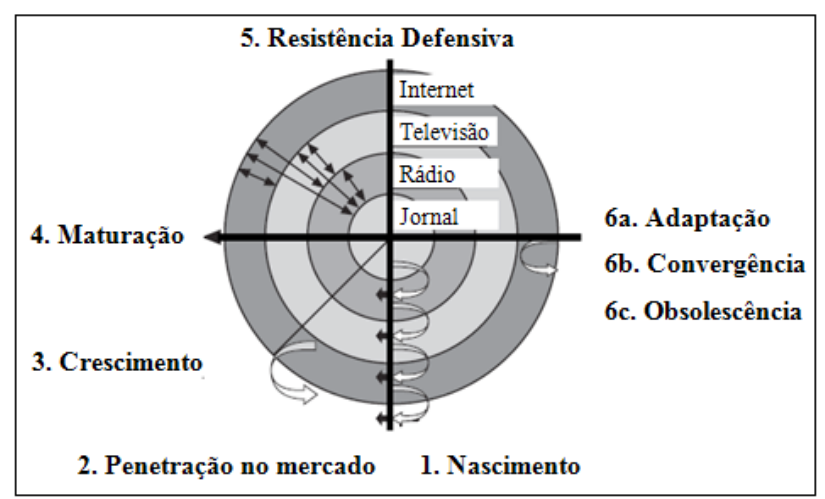

Figura 1 - Modelo de Ciclo de Vida das mídias

Fonte: Traduzida e adaptada de Lehman-Wilzig e Cohen-Avigdor (2004, p. 6).

Lehman-Wilzig e Cohen-Avigdor (2004) explicam que o modelo, que denominam "Ciclo de vida natural da evolução das novas mídias", foi desenhado de forma circular por dois motivos: (1) para indicar a influência da nova 
mídia sobre a velha e, vice-versa, e (2) para sinalizar metaforicamente que cada mídia incorpora elementos (físicos e/ou funcionais) da mídia anterior. Quanto à forma circular, os autores justificam a escolha dizendo que ela thes pareceu bastante adequada para indicar a ordem cronológica de aparecimento das principais mídias e representar o ciclo de vida de uma mídia com seu conjunto de parâmetros, isto é, funções e audiência (LEHMAN-WILZIG; COHEN-AVIGDOR, 2004). Conforme explicam (ver Figura 1), as setas bidirecionais localizadas no quadrante superior esquerdo do círculo indicam que a resistência da mídia mais velha pode começar no estágio de penetração (2), crescimento (3) ou maturação (4), se, e quando, a marca de uma nova mídia aparece, enquanto a mídia mais velha está em um estágio anterior. Os estágios do modelo de Lehman-Wilzig e Cohen-Avigdor (2004) podem ser resumidos conforme segue:

1. Nascimento (invenção tecnológica): representa o início do ciclo de vida; a nova mídia baseia-se em uma tecnologia/mídia existente; seu inventor nem sempre pode prever seu uso real ou final.

2. Penetração no mercado: a nova mídia chega ao mercado, desenvolve novos usos e atrai novos usuários; sua taxa de penetração neste estágio pode chegar a 16\%; se passar desta percentagem, entra no estágio de crescimento, caso contrário, desaparece aos poucos.

3. Crescimento: quando está no estágio de crescimento, a nova mídia tem entre $16 \%$ e $50 \%$ de adoção; seus desenvolvedores e usuários aprendem a explorá-la, aplicam e expandem sua capacidade.

4. Maturação: a nova mídia (ou adaptação da velha mídia) encontra seu lugar no ambiente dinâmico da comunicação; 50 a $90 \%$ das pessoas fazem uso da mídia; é neste estágio que ocorre a máxima utilização e aplicação de suas capacidades.

5. Resistência Defensiva: a competição entre a velha e a nova mídia força a velha mídia a procurar novas direções para preservar sua audiência; o declínio da velha mídia neste estágio oscila entre 90 a 50\%.

6 a. Adaptação: a velha mídia adapta-se a nova situação desenvolvendo diferentes funções e/ou preservando (encontrando) sua (nova) audiência.

6 b. Convergência: a velha mídia não pode sobreviver por si própria, mas preserva sua funcionalidade a fundir-se ou incorporar-se com uma nova mídia.

6 c. Obsolescência: a mídia não se adapta à mudança de forma bem-sucedida; declina ou desaparece.

O modelo de Lehman-Wilzig e Cohen-Avigdor (2004), por uma razão lógica, já que se baseia em estudos anteriores sobre o assunto, é o mais completo de todos. O modelo de Merril e Lowstein e de Saffo, pode-se afirmar que tratam da incorporação de uma nova mídia pela sociedade, respectivamente, focando o fator audiência (penetração por classe social) e o fator tempo. Os estudos de Shaw parecem não prever a adaptação e transformação da mídia, já que ao chegar à velhice, fazendo uso do ciclo de vida humano, chega-se ao fim, a morte, ao desaparecimento. Caspi, Adoni e Nossek, Napoli, O’Brien e Lehman-Wilzig e Cohen-Avigdor preveem em suas teorias e modelos a adaptação e transformação da mídia em busca de uma sobrevida, isto é, visualizam a habilidade de descoberta de novos nichos de 
audiência e novas formas e estilos de apresentação, assim como, capacidade de apresentar seus serviços em novas tecnologias. Parafraseando Fidler (1998, p. 57, tradução nossa), quando emergem novas formas de meios de comunicação, as formas antigas geralmente não morrem, mas continuam evoluindo e adaptando-se.

O que se lerá a seguir é justamente uma resposta da mídia e do mercado às mudanças tecnológicas, sociais e culturais. É uma alternativa de sobrevivência discutida por pesquisadores e profissionais da mídia nos últimos anos nos Estados Unidos, os quais visualizam na distribuição individualizada de conteúdo (notícias e publicidade) a resistência defensiva da indústria tradicional de jornais e revistas.

\section{Adaptação, transformação e evolução das mídias}

Alguns anos atrás, as mídias tradicionais dominavam a produção e publicação de notícias no formato impresso e digital. Este cenário mudou com a popularização da mídia social que permite a qualquer pessoa, com um pouco de tempo e algo para dizer, tornar-se publicador (SCHMIT'T; FIALHO, 2007). Neste contexto, as mídias tradicionais que antes competiam apenas entre si por audiência, passaram a competir também com a mídia social (SCHMITT; FIALHO, 2007).

Desde então, as mídias tradicionais vêm se remodelando para manter a competitividade e atender o consumidor e suas necessidades (SCHMITT; OLIVEIRA, 2009). Recentemente, após anos de pesquisas buscando soluções para a indústria de jornais, ameaçada de extinguir-se devido à diminuição da leitura e, por conseguinte, dos lucros, Peter Vandevanter, vice-presidente da Media Neres Group cunhou o termo notícias individualizadas (HARPER, 2009) e começou a promover

Mídias tradicionais se remodelam para manter a competitividade e atender necessidades do consumidor conferências sobre o assunto. Segundo Vandevanter (2009a, 2009b), o termo consiste na customização em massa da mídia, ou seja, na distribuição de notícias e publicidade baseada nas preferências dos consumidores.

De acordo com Harper (2009), a ideia por trás do conceito é a autosseleção de notícias, entregue em qualquer plataforma e a qualquer hora. No cenário das notícias individualizadas, o assinante escolhe o conteúdo que quer ver e a plataforma em que será ele será apresentado, por exemplo, na tela de um computador, leitor eletrônico (e-reader) ou Personal digital assistants (PDAs) (VANDEVANTER, 2009a).

$\mathrm{Na}$ terceira conferência anual intitulada Individuated News Conference (INC3), realizada em junho de 2009 em Washington, D.C., cinco produtos individualizados (em fase de desenvolvimento e teste) foram apresentados: o I-Newes do Media Neres Groups, uma das maiores empresas de jornais dos Estados Unidos, o jornal customizado do The Washington Times, o Printcas-

\footnotetext{
${ }^{3}$ Syntops é uma empresa alemã, localizada em Augsburg, que fornece toda a solução, de acordo com as necessidades do cliente, para individualizar um jornal ou uma revista. Fornece o portal para o consumidor fazer sua seleção, a tecnologia, a organização da impressão digital e cuida da entrega específica (INDIVIDUALIZE..., 2009).
} 
ting da Knight Foundations, o Personal-Neres da Syntops ${ }^{3}$ e a revista mine da Time Inc.'s. A seghjr, uma descrição de cada produto/projeto apresentado na INC3.

\section{I-News}

No I-Neres, os assinantes podem escolher os tipos de notícias que querem receber e a plataforma: via computador, telefone celular ou uma impressora autônoma conectada a uma linha telefônica. O projeto combina a publicidade com o tipo de conteúdo escolhido pelo assinante (MCCLELLAND, 2009). É um experimento que entrega produtos noticiosos sob medida a baixo custo (SOLOMON, 2009 apud HARPER, 2009).

O Media Neres Groups fornece conteúdo de seus jornais - em Denver, do The Post, e em Los Angeles, do Daily Neres - combinado com notícias da agência The Associated Press, que apresenta notícias em 197 categorias. Desse menu, os assinantes podem construir suas edições customizadas. Uma vez que as notícias são selecionadas pelos leitores, o pacote final é enviado para suas casas via uma impressora inteligente comum sem fio (MCMEEKIN; MOOZAKIS, 2009). Além desse serviço, o Media News Groups lançou um experimento com os hotéis Marriott, em abril de 2009, para a entrega notícias individualizadas aos hóspedes que se hospedam no Residence Inn, no centro de Denver (MCMEEKIN; MOOZAKIS, 2009).

\section{Printcasting}

Printcasting, uma iniciativa da Knight Foundation, permite aos leitores utilizar ferramentas baseadas na web para criar publicações customizadas individuais (MCMEEKIN; MOOZAKIS, 2009). Neste sistema, é dado ao "editor" um menu de conteúdo agregado - de organizações noticiosas, blogueiros e boletins informativos - para que ele escolha o conteúdo que poderá ser utilizado na criação de publicações de nicho, distribuídas digitalmente via e-mail e formato impresso na comunidade local ou locais apropriados, ou publicadas e distribuídas por um jornal qualquer (MCCLELLAND, 2009).

Contribuintes, editores, leitores, anunciantes e distribuidores desempenham os seguintes papéis no Printcasting, segundo McClelland (2009):

1. Contribuintes: fornecem conteúdo regularmente atualizado, que pode ser acessado através de um feed (alimentador web) - por exemplo, blogueiros que querem expandir sua audiência e ganhar receitas adicionais.

2. Editores: escolhem vários feeds, montam e enviam publicações de nicho.

3. Leitores: podem baixar a publicação - ao fazerem isto, fornecem algum tipo de informação demográfica para os anunciantes em potencial;

4. Anunciantes: colocam seus anúncios on-line.

O Princasting conta ainda com o recurso revisar e aprovar, que assegura a qualidade do produto, uma vez que é permitido ao editor por meio de tal recurso apresentar uma última edição da publicação no site (MCCLELLAND, 2009). A Figura 2 mostra como o Printcasting funciona: 


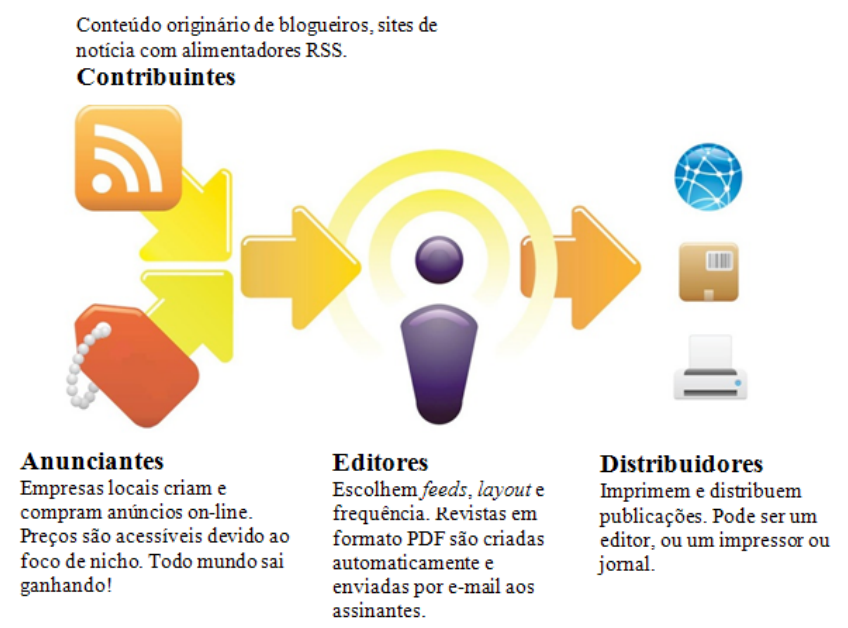

Figura 2 - Funcionamento do Princasting

Fonte: Traduzido de Pacheco $(2009$, p. 8).

\section{PersonalNews}

O PersonalNerws foi a primeira implementação que ofereceu jornal individual personalizado (DORSCH, 2009). Seus experimentos iniciaram em 8 de dezembro de 2008 (MALERIUS, 2009; PERSONALNEWS..., 2009b). Os idealizadores pretendiam transformá-lo em um modelo de negócio sério e sustentável, com cooperação estreita dos editores participantes (PERSONALNEWS..., 2009b). O PersonalNeres, em sua fase experimental, permitia aos leitores criar seu próprio jornal, através de um portal de internet que possibilitava combinar seções específicas de jornais nacionais e internacionais (DORSCH, 2009; MCMEEKIN; MOOZAKIS, 2009; PERSONALNEWS..., 2009a; PERSONALNEWS..., 2009b). O projeto piloto da Syntops foi conduzido na Suíça, onde a Syntops testou o jornal em cooperação com o correio suíço, responsável pela impressão e entrega do jornal final (DORSCH, 2009; MCMEEKIN; MOOZAKIS, 2009). Na Suíça, o PersonalNeres foi entregue aos assinantes em dois formatos: PDF e impresso (MCMEEKIN; MOOZAKIS, 2009). As cópias, impressas em preto e branco, tamanho A3, foram entregues cinco dias por semana, de segunda a sexta-feira, para 100 assinantes de uma região selecionada (MALERIUS, 2009; PERSONALNEWS..., 2009b). Enquanto o fornecimento do serviço foi perfeito tecnica e logicamente, a versão impressa simplesmente não manteve o ritmo da versão eletrônica em termos de interesse atual e custo (PERSONALNEWS..., 2009b). A proposta do PersonalNeres, distribuído gratuitamente, foi demonstrar a viabilidade técnica de um jornal customizado (PERSONALNEWS..., 2009b). No projeto piloto, os leitores puderam escolher seu conteúdo preferido através da seleção de categorias e seções específicas de aproximadamente 20 jornais nacionais e internacionais (PERSONALNEWS..., 2009b). Cada cópia eletrônica do jornal dependeu de um sofisticado sistema e software desenvolvido ao longo de quatro anos pela fabricante de software Syntops $\mathrm{GmbH}$. O software foi projetado para lidar com cada aspecto do processo do momento que o leitor coloca sua solicitação para a recuperação, passando pela preparação e identificação de conteúdo relevante, até a customização 
e a ativação controlada das máquinas de impressão digital (PERSONALNEWS..., 2009b).

Segundo Dorsch (2009), com o PersonalNews objetiva-se:

1. aumentar a circulação do jornal impresso;

2. motivar novos grupos de leitores a comprar jornais;

3. reconquistar leitores que estavam migrando para novas mídias;

4. estender a cobertura de títulos de jornais nacionais e internacionais;

5. abrir e explorar o marketing direto do mercado publicitário ${ }^{4}$;

6. coletar dados do leitor/usuário e de seu comportamento de uso de forma precisa;

7. obter atualização de vendas por minuto e análises de distribuição.

\section{The Washington Times}

Durante quatro semanas (entre maio e junho de 2009), The Washington Times testou um projeto inovador em que 60 assinantes acessaram uma página hospedada no Syntops para escolher, de um menu de opções, os tipos e a quantidade de conteúdo que desejavam receber (SLEVIN, 2009; MCMEEKIN; MOOZAKIS, 2009). Este projeto seguiu o mesmo conceito do PersonalNeres, mas com conteúdo de um único editor (DORSCH, 2009).

As edições customizadas ou individualizadas variaram de 20 a 60 páginas e foram entregues por e-mail aos assinantes (SLEVIN, 2009; MCMEEKIN; MOOZAKIS, 2009). Com base nas preferências pessoais, os participantes do projeto puderam facilmente aumentar ou diminuir o número de matérias políticas, nacionais, de segurança nacional e cultura em sua edição individualizada impressa. Também foi permitido que selecionassem colunistas de opinião pelo nome e adicionassem conteúdo normalmente não publicado no semanário, como esportes e outras colunas (SLEVIN, 2009).

No The Washington Times foi implementada a abordagem single publisher, em português, único editor, que deixava os leitores escolherem do conteúdo existente no jornal o que eles desejavam ler (INDIVIDUALIZE..., 2009). Segundo a empresa Syntops (INDIVIDUALIZE..., 2009), previa-se que tal abordagem pudesse abrir novos e adicionais mercados de publicidade, como: mala direta, publicidade segmentada, publicação coorporativa individualizada e publicidade pull. Contudo, primeiramente, foi implementada para individualizar módulos, como metade ou um quarto das páginas (INDIVIDUALIZE..., 2009).

\section{mine}

A luxuosa fabricante de carros Lexus associou-se a Time Inc. e a American Express Publishing Corporation para produzir a revista mine, disponibilizada em formato impresso e on-line (ADAIR; CONNELL; HUBBELL,

\footnotetext{
${ }^{4}$ Segundo Dorsch (2009), o sistema de produção customizada abre novos mercados publicitários, já que a publicidade também pode ser entregue baseada nos gostos e preferências do leitor/usuário.
} 
2009). Lançada em abril de 2009, mine, que carrega o slogan "my magazine, may way", convida os leitores a criar uma revista gratuita, pessoal e customizada, com conteúdo editorial de cinco dos oito títulos selecionados: TIME, Sports Illustrated, Food E Wine, Money, Golf, Real Simple, InStyle e Travel + Leisure (MOOZAKIS, 2009; ADAIR; CONNELL; HUBBELL, 2009).

O projeto antevia que a revista customizada fosse entregue no formato impresso por correio ou no formato digital, via web ou telefone celular, a cada duas semanas, por 10 semanas, aos participantes do projeto (leitores que optaram pelo serviço) (MOOZAKIS, 2009; ADAIR; CONNELL; HUBBELL, 2009). Os leitores também podiam receber alerta de notícias, informação e entretenimento através de um widget (componente de interface gráfica do usuário) customizado ou de uma aplicação móvel enviada via alimentadores RSS (Really Simple Syndication) diretamente para seu site pessoal, rede social ou aparelho sem fio (ADAIR; CONNELL; HUBBELL, 2009).

Segundo Adair, Connell e Hubbell (2009), com base nos interesses e nas paixões do leitor, e curada pelos editores da Time Inc. e American Express Publishing, mine é um exemplo na produção de revista e interação com o leitor/usuário. Em 36 páginas, incluindo quatro anúncios de página simples para o Lexus RX, mine apresentou a melhor das melhores (ADAIR; CONNELL; HUBBELL, 2009). Além do conteúdo editorial customizado, a meta da Lexus era também personalizar a publicidade com base na localização geográfica e na preferência de conteúdo, o que levaria a criação de mais de um milhão de edições exclusivas da mine (ADAIR; CONNELL; HUBBELL, 2009).

Todos os produtos mencionados mostram que os veículos tradicionais estão se remodelando, criando novos produtos e novas parcerias para que as velhas e as novas mídias coexistam, interajam e se complementem, até que, conforme Briggs e Burke (2006), se estabeleça alguma divisão de trabalho ou função. A entrega de conteúdo individualizado é uma estratégia que tem o potencial para salvar a indústria tradicional de comunicação.

\section{Considerações finais}

Em uma Era de constantes avanços tecnológicos é extremamente difícil prever mudanças exatas. Como disse Nielsen (2000, p. 376), “[a] única previsão garantida é que a única constante é a mudança.”

O processo de comunicação mudou drasticamente com a internet e as novas mídias. Elas colocaram em xeque a existência das mídias tradicionais como mediadoras do conhecimento e das informações na sociedade. $\mathrm{Na}$ atualidade, percebe-se a coexistência entre as velhas e as novas mídias e a convergência de formatos, assim como um grande esforço (resistência defensiva) por parte das mídias e veículos tradicionais do conhecimento para manter a competitividade, a audiência ou atrair novos usuários diante das novas tecnologias e formas de comunicação.

No começo deste século, Briggs e Burke (2006) comentavam que a mídia precisava ser vista como um sistema, um sistema em contínua mudança. Pare- 
ce que essa é a visão dos idealizados dos produtos individualizados apresentados neste trabalho: a velha e a nova mídia estão se adaptando, transformando e evoluindo, bem como se complementando. Se os produtos individualizados passarem da fase de penetração e crescimento, eles chegarão ao estágio de adaptação do modelo de desenvolvimento proposto por Lehman-Wilzig e Cohen-Avigdor, estágio que prevê uma adaptação da velha mídia no mercado, não somente o estágio de adaptação de uma nova mídia.

Acredita-se que - diferente dos papiros, tambores, tochas e rolos de pergaminhos que caíram em desuso, obsolescência - as mídias tradicionais (jornais, revistas, rádio e TV) seguirão se reinventando, reconstruindo seus modelos de negócios, seus formatos e suas funções na Era da Informação e do Conhecimento, pelo menos por um bom tempo.

\section{Referências bibliográficas}

ADAIR, Daphne; CONNEL, Kris; HUBBELL; Nancy. Beyond mp3 Players: Creating A Magazine Playlist. [s.n.], Torrance, CA; New York, NY, 18 mar. 2009. Disponível em: <http://newsandtechinc3.wordpress.com>. Acesso em: 26 jul. 2009.

BRIGGS, Asa; BURKE, Peter. Uma história social da mídia: de Gutenberg à Internet. Trad. Maria Carmelita Pádua Dias. 2 ed. rev. e ampl. Rio de Janeiro: Jorge Zahar Ed., 2006. 375p.

CASTELLS, Manoel. A galáxia da internet: reflexões sobre a internet, os negócios e a sociedade. Trad. Maria Luiza X. de A. Borges; revisão Paula Vaz. Rio de Janeiro: Jorge Zahar, 2003.

DORSCH, Gregor. Mass-customization for print media industry. Washington, D.C., [s.n.], 2009. 21 slides. Color. Acompanha texto. Disponível em: <http://newsandtechinc3.wordpress.com>. Acesso em: 26 jul. 2009.

FIDLER, Roger. Mediamorfosis: comprender los nuevos medios. Buenos Aires, Argentina: Granica, 1998. 440 p.

HARPER, Jennifer. Newspaper developing 'individuated news'. The Washington Times. Washington, D.C., 25 june 2009. Disponível em: <http:// www.washingtontimes.com/news/2009/jun/25/newspaper-executives-takereaders-personally/>. Acesso em: 27 jul. 2009.

INDIVIDUALIZE your own publications! Syntops GmbH. [s.d.]. Disponível em: <http://syntops.de/index.php?id=63\&L=3>. Acesso em: 08 set. 2009.

JENKINS, Henry. Cultura da Convergência. Trad. Susana Alexandria. São Paulo: Aleph, 2008.

LEHMAN-WIZIG, Sam; COHEN-AVIGDOR, Nava. The natural life cycle of new media evolution. New Media \& Society, London, Thousand Oaks, CA and New Delhi, v. 6, n. 6, p. 707-730, dez. 2004.

MACHLUP, Fritz. The production and distribution of knowledge in The United States. Princeton, New Jersey: Princeton University Press, 1962. 416 p.

MALERIUS, Frank. Washington and Zofingen over breakfast. [press business]. Hamburg, Germany: Presse Fachverlag, n. 1, p. 34-35, 2009. Disponível 
em: <http://www.press-business.com/pdf/2009_01_press_business.pdf>. Acesso em: 8 set. 2009.

MCCLELLAND, Pat. The Individuated Newspaper: newspaper of the future. Media Update. 2 Abril 2009. Disponível em: < http://www.mediaupdate. co.za>. Acesso em: 26 jul. 2009.

MCMEEKIN, Tara; MOOZAKIS, Chuck. Products show worth at INC3. In: News on Demand. Denver, Colo: Newspapers \& Technology, n. 2, Supplement Issue, p. 3-4 e 6, jul. 2009. Disponível em: <http://www2.newsandtech.com/special_edition/?pub=Special\%20Section:News\%20On\%20Demand\%20Supplement $>$. Acesso em: 27 jul. 2009.

MEDITSCH, Eduardo. O Jornalismo é uma Forma de Conhecimento? BOCC. Biblioteca On-line de Ciências da Comunicação, Lisboa, [s.v., s. n.], p. 1-13, 1997. Disponível em: < http://www.bocc.uff.br/pag/meditsch-eduardo-jornalismo-conhecimento.pdf $>$. Acesso em: 10 mar. 2010.

MOOZAKIS, Chuck. Time sets next step for 'mine'. In: News on Demand. Denver, Colo: Newspapers \& Technology, n. 2, Supplement Issue, p. 3-4 e 6, jul. 2009. Disponível em: <http://www.newsandtech.com/NewsonDemand0709.pdf>. Acesso em: 27 jul. 2009.

NIELSEN, J. Projetando Websites. Tradução de Ana Gibson. Rio de Janeiro: Campus, 2000.

PACHECO, Dan. Princasting: People-Powered Magazines. Washington, D. C.: [s.d.], 2009, 28 slides, color. Acompanha texto. In: INVIDUATED NEWS CONFERENCE, 3, 2009a, Washington, D.C. Disponível em: < http://newsandtechinc3.wordpress.com>. Acesso em: 26 jul. 2009.

PERSONALNEWS - your daily individual newspaper. Syntops GmbH. [s.d.]. Disponível em: <http://syntops.de/index.php? id=63\&L=3>. Acesso em: 08 set. $2009 \mathrm{~b}$.

PERSONALNEWS: you decide what will feature in tomorrow's newspaper. PersonalNews. [s.d.]. Disponível em: <https://personalnews.syntops.de/index.php? id=10\&no_cache $=1 \& \mathrm{~L}=5>$. Acesso em: 30 ago. 2009a.

SAAD, B. Estratégias para a mídia digital. São Paulo: Senac, 2003.

SCHMITT, Valdenise. Comunicação Digital: Produção Jornalística na Era dos bits e bytes. 2003. 75f. Monografia. (Especialização em Novas Mídias, Rádio e TV) - Universidade Regional de Blumenau, Blumenau, 2003.

SCHMITT, Valdenise; FIALHO, Francisco Antonio Pereira. A Cauda Longa e o Jornalismo: como a teoria da Cauda Longa se aplica ao jornalismo. Revista Fronteiras, São Leopoldo, v. IX, n. 3, p. 197-204, 2007.

SCHMITT, Valdenise; OLIVEIRA, Leonardo Gomes. Personalização de Notícias: uma edição para cada leitor. Eptic On-Line (UFS), v. XI, p. 1-18, 2009.

SLEVIN, Jonathan. Washington Times produces 'Individuated' National Weekly: Print edition was published on a high-speed, ink-jet press. The Washington Times, Washington, D.C., [s.d.]. Disponível em: <http://newsandtechinc3.wordpress.com >. Acesso em: 26 jul. 2009.

STANOEVSKA-SLABEVA, Katarina: The Concept of Knowledge Media: The Past and the Future. In: GRÜTTER, Rolf (Org.). Knowledge Media in Healthcare: Opportunities and Challenges. Hershey, USA: Idea Group Publishing, 2002, p. 1-16. 
VANDEVANTER, Peter. At long last the personal newspaper is here: it's green, targeted and multi-platform. In: INVIDUATED NEWS CONFERENCE, 3, 2009a, Washington, D.C. Disponível em: <http://newsandtechinc3.wordpress.com>. Acesso em: 26 jul. 2009.

VANDEVANTER, Peter. At long last, the Personal "Newspaper" has arrived: It's Green, Targeted and Multi-platform. Washington, D.C., [s.n.], 2009b. 15 slides. Color. Acompanha texto. Disponível em: <http://newsandtechinc3.wordpress.com>. Acesso em: 26 jul. 2009. 